\title{
Estimativas de parâmetros genéticos e métodos de seleção para o melhoramento genético de Pinus oocarpa Schiede(1)
}

\author{
Paulo de Tarso Barbosa Sampaio(2), Marcos Deon Vilela de Resende ${ }^{(3)}$ e Antonio José de Araújo(4)
}

Resumo - Este trabalho teve por objetivo estudar a variabilidade genética entre e dentro de procedências e progênies e determinar o ganho genético em volume de madeira, em Pinus oocarpa Schiede. $\mathrm{O}$ experimento foi instalado em Angatuba, SP, em delineamento de blocos de famílias compactas, com nove repetições e parcelas lineares de seis plantas. A produtividade volumétrica média obtida de sete procedências foi de $0,296 \mathrm{~m}^{3}$ de madeira por árvore aos nove anos de idade. Para estabelecer um pomar de sementes por mudas, a seleção (no bloco) de 189 árvores ( 27 por procedência) com os maiores diâmetros na altura do peito (DAP) permite aumentos na produtividade volumétrica de $0,327 \mathrm{~m}^{3}$, $0,338 \mathrm{~m}^{3}$ e $0,341 \mathrm{~m}^{3}$ por árvore, na seleção individual, combinada e índice multiefeito, respectivamente. Em pomar de sementes clonais, a seleção (no experimento) de 28 árvores (4 por procedência) com maiores DAPs permite aumentos na produtividade volumétrica de $0,345 \mathrm{~m}^{3}, 0,355 \mathrm{~m}^{3}$ e $0,356 \mathrm{~m}^{3}$, por árvore, na seleção individual, combinada e índice multiefeito, respectivamente.

Termos para indexação: índice de seleção, variação genética, ganho genético, valor genético.

Estimates of genetic parameters and selection methods for genetic improvement of Pinus oocarpa Schiede

\begin{abstract}
This work aimed to study the genetic variability within and between provenances and progenies and to determine the genetic gain related to timber volume, in Pinus oocarpa. The experiment was carried up in Angatuba, São Paulo, Brazil, in a compact family block design, with nine replications and six plant plots. The results of seven provenances showed a production of $0.296 \mathrm{~m}^{3}$ of timber per tree at nine years-old. To establish seedling seed orchard, 189 trees with the greatest genetic gain in diameter at breast height ( 27 trees per provenance) were selected. The selection of these trees will allow an increase in the current volumetric timber production, reaching $0.327 \mathrm{~m}^{3}$ per tree (individual selection), $0.338 \mathrm{~m}^{3}$ per tree (combined selection) and $0.341 \mathrm{~m}^{3}$ per tree (multi-effect index). To implement a clonal seed orchard, 28 trees with the greatest genetic gain in diameter at breast height (four trees per provenance) were selected. The selection of these trees will allow an increase in the current volumetric timber production, reaching $0.345 \mathrm{~m}^{3}$ per tree (individual selection), $0.355 \mathrm{~m}^{3}$ per tree (combined selection) and $0.356 \mathrm{~m}^{3}$ per tree (multi-effect index).
\end{abstract}

Index terms: selection index, genetic variation, genetic gain, breeding value.

(1) Aceito para publicação em 17 de agosto de 2001

Extraído da tese de doutorado apresentada pelo primeiro autor à Universidade Federal do Paraná (UFPR), Curitiba, PR.

(2) Instituto Nacional de Pesquisas da Amazônia, Coordenação de Pesquisas de Silvicultura Tropical, Caixa Postal 478 , CEP 69011-970 Manaus, AM. E-mail: sampaio@inpa.gov.br

(3)Embrapa-Centro Nacional de Pesquisa de Florestas, Caixa Postal 319, CEP 83411-000 Colombo, PR. E-mail deon@cnpf.embrapa.br

(4) Universidade Estadual do Centro Oeste, Campus de Irati, Caixa Postal 730, CEP 85015-430 Guarapuava, PR. E-mail: dearaujo@onda.com.br

\section{Introdução}

Os programas tradicionais de melhoramento genético no Brasil têm se fundamentado principalmente na seleção entre e dentro de famílias (seleção individual). Nesse esquema, identificam-se, em uma primeira etapa, as melhores famílias com base na média das parcelas. No caso de famílias de meios-irmãos, são explorados apenas $25 \%$ da variância aditiva total na seleção entre famílias. Na etapa seguinte, selecionam-se, nas famílias superiores, as plantas de 
melhor desempenho. Essa prática proporciona ganhos adicionais, por explorar a fração da variância utilizada na seleção entre famílias, mas apresenta o inconveniente de se basear em valores fenotípicos individuais, sujeitos a maior influência dos efeitos ambientais (Falconer, 1987).

A seleção combinada constitui-se em procedimento biométrico de identificação de genótipos, baseado em um índice que leva em consideração, simultaneamente, o comportamento de indivíduos e de sua família. Assim, dependendo dos pesos de ponderação do indivíduo e da família, seria possível, pelo uso da seleção combinada, selecionar aquelas plantas superiores, porém de famílias de desempenho médio, ou aquelas plantas de desempenho médio pertencentes às famílias superiores (Falconer, 1987). Neste método, a seleção é baseada em medidas genéticas (valores genéticos líquidos) e não fenotípicas dos candidatos à seleção.

A melhor estimativa do valor genético de um indivíduo candidato à seleção corresponde ao produto do valor fenotípico desse indivíduo pela regressão do genótipo sobre o fenótipo (Falconer, 1987). Dentro desse conceito enquadram-se todas as modalidades de seleção baseadas em índices, sejam índices univariados ou multivariados (Resende \& Higa, 1994b). Quando a seleção é praticada com base em um índice, combinando uma série de informações referentes aos indivíduos candidatos à seleção, esperam-se respostas máximas nos valores genéticos desses indivíduos e, conseqüentemente, maior precisão na seleção.

A seleção com base nos testes de progênies utiliza, geralmente, apenas duas fontes de informações: o desvio do valor individual em relação à média da família no bloco, e o desvio da média da família em relação à média geral do teste. $\mathrm{O}$ emprego destas duas fontes permite a aplicação dos métodos de seleção individual e seleção combinada (Resende \& Higa, 1994b). Entretanto, em esquemas de melhoramento, sem o emprego de sementes remanescentes e com baixo número de plantas por parcela nos experimentos, utilizando-se apenas estas duas fontes de informações, percebe-se que frações da variância genética aditiva não são consideradas na seleção, pois são retidas no efeito de parcelas e de blocos, efeitos esses que também propiciam correção para efeitos ambientais. A seleção pelo índice multiefeito, por considerar esses efeitos, possibilita a maximização da precisão na seleção.

Este trabalho objetivou estudar a grandeza da variação genética e estimar os parâmetros genéticos relacionados aos caracteres de crescimento e densidade básica da madeira das árvores das populações de $P$. oocarpa, determinar o ganho genético em volume de madeira por meio da seleção individual, combinada e pelo índice multiefeito na instalação de pomar de sementes por mudas e pomar de sementes clonais.

\section{Material e Métodos}

A relação das procedências e das testemunhas, número de progênies de meios-irmãos e a localização geográfica são apresentados na Tabela $1 . \mathrm{O}$ experimento foi plantado em Angatuba, SP, a $23^{\circ} 29^{\prime} \mathrm{S}, 48^{\circ} 25^{\prime} \mathrm{W}$ e $900 \mathrm{~m}$ de altitude, em uma região com precipitação média anual de $1.190 \mathrm{~mm}$, com temperatura mínima e máxima de $14^{\circ} \mathrm{C}$ e $21^{\circ} \mathrm{C}$, respectivamente, solo do tipo RPV-RLV (Regossolo em transição para Podzólico Vermelho-Amarelo, grupamento indiscriminado).

O solo foi preparado com aração e gradeação e o plantio efetuado em março de 1984, no espaçamento 3 x $3 \mathrm{~m}$. O delineamento utilizado foi o de blocos de famílias compactas, com nove repetições e parcelas lineares de seis plantas. As medições foram realizadas aos nove anos de idade. Os dados de volume (VOL), forma do fuste (FF) e densidade básica da madeira (DEN) foram obtidos em nível de plantas individuais, ao passo que para copa quebrada (CQ) e sobrevivência, os dados foram obtidos em nível de médias de parcelas de seis plantas. Para o caráter forma do fuste, foi utilizado critério subjetivo e avaliação visual, atribuindo-se notas de 1 (pior) a 3 (melhor).

As análises dos caracteres mensurados foram realizadas com uso do programa SELEGEN (Resende et al., 1994a), obedecendo o seguinte modelo estatístico:

$\mathrm{Y}_{\mathrm{ijkl}}=\mu+\mathrm{p}_{\mathrm{i}}+\mathrm{f}_{\mathrm{j}(\mathrm{i})}+\mathrm{b}_{\mathrm{k}}+\mathrm{pb}_{\mathrm{ik}}+\mathrm{fb}_{\mathrm{j}(\mathrm{i}) \mathrm{k}}+\mathrm{d}_{(\mathrm{ijk} \mathrm{k})}$,

$\mathrm{Y}_{\mathrm{ijkl}}=$ observação da planta 1 , da procedência $\mathrm{i}$, da progênie $\mathrm{j}$, na repetição $\mathrm{k}$;

$\mu=$ média geral da população;

$\mathrm{p}_{\mathrm{i}}=\mathrm{o}$ efeito da procedência $\mathrm{i}$, com $\mathrm{i}=1,2,3 \ldots 7$;

$f_{j(i)}=$ efeito da progênie $j$, dentro da procedência $i$;

$\mathrm{b}_{\mathrm{k}}=$ efeito do bloco $\mathrm{k}$; com $\mathrm{k}=1,2,3 \ldots 9$;

$\mathrm{pb}_{\mathrm{ik}}=$ interação da procedência i, com o bloco k;

$\mathrm{fb}_{\mathrm{j}(\mathrm{i}) \mathrm{k}}=$ interação da família j, dentro da procedência i, com o bloco k; 
$\mathrm{d}_{(\mathrm{ijkl})}=$ efeito entre plantas dentro de parcela, associado ao indivíduo 1 , da progênie $\mathrm{j}$, da procedência i, na repetição $\mathrm{k}$, com $1=1,2, \ldots .6$.

O esquema de análise de variância referente aos blocos de famílias compactas, em médias de parcelas, com as respectivas esperanças dos quadrados médios, considerando todos os efeitos como aleatórios, exceto a média, seguiu o modelo sugerido por Resende et al. (1994) (Tabela 2).

\section{Estimativa dos coeficientes de herdabilidade}

Os coeficientes de herdabilidade no sentido restrito de indivíduo na parcela $\left(\hat{\mathrm{h}}_{\mathrm{d}}^{2}\right)$, herdabilidade no sentido restrito de progênie ( $\left.\hat{\mathrm{h}}_{\mathrm{f}}^{2}\right)$, herdabilidade no sentido restrito de parcela $\left(\hat{\mathrm{h}}_{\mathrm{p}}^{2}\right)$, herdabilidade no sentido restrito de blocos $\left(\hat{h}_{b}^{2}\right)$, herdabilidade no sentido restrito de indivíduo no bloco $\left(\hat{\mathrm{h}}_{\mathrm{ib}}^{2}\right)$, herdabilidade no sentido restrito de plantas individuais no experimento $\left(\hat{\mathrm{h}}_{\mathrm{ie}}^{2}\right)$ associado aos diferentes efeitos do modelo linear dentro de cada procedência e do erro associado à estimativa $\mathrm{s}\left(\hat{\mathrm{h}}_{\overline{\mathrm{F}}}^{2}\right)$, foram estimados segundo Resende \& Higa (1994b).

\section{Estimativa de correlações genéticas e fenotípicas}

As correlações genéticas de indivíduos e fenotípicas em nível de médias de famílias foram estimadas a partir das seguintes expressões (Zobel \& Talbert, 1984; Vencovsky \& Barriga, 1992):

a) Coeficiente de correlação genética aditiva $\left(\mathrm{r}_{\mathrm{A}}\right)$

$\mathrm{r}_{\mathrm{Axy}}=\frac{\operatorname{CôV}_{\mathrm{A}}(\mathrm{x}, \mathrm{y})}{\left(\hat{\sigma}_{\mathrm{Ax}}^{2} \cdot \hat{\sigma}_{\mathrm{Ay}}^{2}\right)^{1 / 2}}$

$\hat{\sigma}_{\mathrm{Ax}}^{2}=$ variância genética aditiva do caráter $\mathrm{x}$,

$\hat{\sigma}_{\mathrm{Ay}}^{2}=$ variância genética aditiva do caráter $\mathrm{y}$. b) Coeficiente de correlação em médias de progênies $\left(\mathrm{r}_{\overline{\mathrm{F}}}\right)$ $\mathrm{r}_{\overline{\mathrm{F}}}=\frac{\mathrm{COO} \mathrm{V}_{\overline{\mathrm{F}}}(\mathrm{x}, \mathrm{y})}{\left(\hat{\sigma}_{\overline{\mathrm{Fx}}}^{2} \cdot \hat{\sigma}_{\overline{\mathrm{Fy}}}^{2}\right)^{1 / 2}}$

$\hat{\sigma}_{\overline{\mathrm{Fx}}}^{2}=$ variância fenotípica do caráter $\mathrm{x}$, em médias de famílias,

$\hat{\sigma}_{\overline{\mathrm{Fy}}}^{2}=$ variância fenotípica do caráter y, em médias de famílias.

c) Desvio-padrão do coeficiente de correlação genética aditiva (Falconer, 1987)

$S\left(r_{\text {Axy }}\right)=\frac{1-\left(\mathrm{r}_{\text {Axy }}\right)^{2}}{\sqrt{2}}\left[\frac{s\left(\hat{\mathrm{h}}_{\mathrm{fx}}^{2}\right)+\mathrm{s}\left(\hat{\mathrm{h}}_{\mathrm{fy}}^{2}\right)}{\hat{\mathrm{h}}_{\mathrm{fx}}^{2} \cdot \hat{\mathrm{h}}_{\mathrm{fy}}^{2}}\right]^{1 / 2}$

$\mathrm{S}\left(\mathrm{r}_{\mathrm{Axy}}\right)=$ desvio-padrão do coeficiente de correlação genética aditiva dos caracteres x e y;

$\mathrm{r}_{\mathrm{Axy}}=$ coeficiente de correlação genética aditiva entre $\mathrm{x}$ e $\mathrm{y}$; $\mathrm{s}\left(\hat{\mathrm{h}}_{\mathrm{fx}}^{2}\right)=$ desvio-padrão do coeficiente de herdabilidade em famílias do caráter $\mathrm{x}$;

$s\left(\hat{\mathrm{h}}_{\mathrm{fy}}^{2}\right)=$ desvio-padrão do coeficiente de herdabilidade em famílias do caráter y;

$\hat{\mathrm{h}}_{\mathrm{fx}}^{2}=$ coeficiente de herdabilidade do caráter $\mathrm{x}$, em famílias; $\hat{\mathrm{h}}_{\mathrm{fy}}^{2}=$ coeficiente de herdabilidade do caráter y, em famílias.

\section{Progresso genético}

As expressões para estimar o progresso genético com seleção individual, adequada para experimentos com várias plantas por parcela, foi apresentada por Resende \& Higa (1994a)

$I=\hat{h}_{\mathrm{ie}}^{2}\left(X_{\mathrm{ijk}}-\bar{X}_{\ldots} ..\right)$ e $\hat{\mathrm{h}}_{\mathrm{ib}}^{2}\left(\mathrm{X}_{\mathrm{ijk}}-\bar{X}_{\cdot \mathrm{j}}\right)$

$\mathrm{I}=$ valor genético;

$\hat{\mathrm{h}}_{\mathrm{ie}}^{2}=$ coeficiente de herdabilidade no sentido restrito de indivíduos no experimento;

Tabela 1. Caracterização das regiões de coleta das procedências de $P$. oocarpa e testemunhas testadas em Angatuba, SP.

\begin{tabular}{|c|c|c|c|c|c|}
\hline Procedência & $\begin{array}{l}\text { Progênies } \\
\left(\mathrm{n}^{\mathrm{o}}\right)\end{array}$ & Latitude & Longitude & $\begin{array}{l}\text { Altitude } \\
\text { (m) }\end{array}$ & $\begin{array}{c}\text { Precipitação } \\
(\mathrm{mm})\end{array}$ \\
\hline San Luís Jilotepeque-Guatemala & 8 & $14^{\circ} 37^{\prime} \mathrm{N}$ & $89^{\circ} 46^{\prime} \mathrm{W}$ & $950-1.010$ & 895 \\
\hline El Castaño - Guatemala & 8 & $15^{\circ} 1^{\prime} \mathrm{N}$ & $90^{\circ} 9^{\prime} \mathrm{W}$ & $930-1.330$ & $900^{(1)}$ \\
\hline Tablazon - Honduras & 6 & $14^{\circ} 9^{\prime} \mathrm{N}$ & $87^{\circ} 37^{\prime} \mathrm{W}$ & $960-1.120$ & 1.548 \\
\hline Guaimaca - Honduras & 8 & $14^{\circ} 32^{\prime} \mathrm{N}$ & $86^{\circ} 48^{\prime} \mathrm{W}$ & $800-1.040$ & 1.250 \\
\hline San Marcos - Honduras & 8 & $13^{\circ} 24^{\prime} \mathrm{N}$ & $86^{\circ} 51^{\prime} \mathrm{W}$ & $1.040-1.200$ & 877 \\
\hline Las Crucitas - Honduras & 8 & $14^{\circ} 7^{\prime} \mathrm{N}$ & $86^{\circ} 37^{\prime} \mathrm{W}$ & $1.010-1.110$ & 1.169 \\
\hline La Campa - Honduras & 8 & $14^{\circ} 28^{\prime} \mathrm{N}$ & $88^{\circ} 35^{\prime} \mathrm{W}$ & $1.110-1.405$ & 1.550 \\
\hline CO 202-(M P R) - Belize ${ }^{(2)}$ & - & $17^{\circ} 0^{\prime} \mathrm{N}$ & $88^{\circ} 55^{\prime} \mathrm{W}$ & 700 & - \\
\hline BR 405 - Agudos, Brasil ${ }^{(3)}$ & - & $22^{\circ} 22^{\prime} \mathrm{S}$ & $48^{\circ} 52^{\prime} \mathrm{W}$ & 550 & 1.523 \\
\hline
\end{tabular}

${ }^{(1)}$ Valores estimados. ${ }^{(2)}$ P. tecunumanii. ${ }^{(3)}$ P. oocarpa 
$\hat{\mathrm{h}}_{\mathrm{ib}}^{2}=$ coeficiente de herdabilidade no sentido restrito de indivíduos no bloco;

$\mathrm{X}_{\mathrm{ijk}}=$ valor do indivíduo $\mathrm{k}$ na parcela $\mathrm{ij}$;

$\overline{\mathrm{X}}_{. .}=$média geral do experimento;

$\overline{\mathrm{X}}_{. \mathrm{j} .}=$ média do bloco.

O progresso genético com seleção combinada é estimada pelo índice

$\mathrm{I}=\hat{\mathrm{h}}_{\mathrm{d}}^{2} \mathrm{X}_{\mathrm{ijk}}-\hat{\mathrm{h}}_{\mathrm{d}}^{2}-\overline{\mathrm{X}}_{\mathrm{ij} .}+\hat{\mathrm{h}}_{\mathrm{f}}^{2}-\overline{\mathrm{X}}_{\mathrm{i} . .}-\hat{\mathrm{h}}_{\mathrm{f}}^{2}-\overline{\mathrm{X}} \ldots$,

que corresponde à multiplicação dos valores fenotípicos referente ao indivíduo, média de parcela, média da família e média geral do experimento pelas respectivas herdabilidades, onde:

$\mathrm{I}=$ valor genético;

$\mathrm{X}_{\mathrm{i}}=$ média da família;

$\overline{\mathrm{X}}_{\mathrm{ij.} .}=$ média da parcela;

$\overline{\mathrm{X}}_{\ldots}=$ média geral do experimento;
$\mathrm{X}_{\mathrm{ijk}}=$ desvio do valor individual (parcela);

$\hat{\mathrm{h}}_{\mathrm{d}}^{2}=$ herdabilidade no sentido restrito associado aos efeitos de indivíduos dentro de parcelas;

$\hat{\mathrm{h}}_{\mathrm{f}}^{2}=$ herdabilidade no sentido restrito de progênies.

A seleção índice multiefeito baseia-se na multiplicação dos valores fenotípicos referentes a indivíduo, média de parcela, média de família, média de bloco e média geral do experimento pelos coeficientes de ponderação dos índices (herdabilidades).

Os coeficientes de ponderação dos índices são determinados de forma que a correlação entre o índice e o valor genético seja máxima. Essa maximização ocorre pela regressão do valor genético sobre os valores fenotípicos, o que conduz a um sistema matricial (Resende et al., 1994).

A expressão para estimação do progresso genético com seleção baseada em desvios, adequada para experimentos com várias plantas por parcela, foi deduzida por Resende \& Higa (1994a):

Tabela 2. Esquema de análise de variância para o modelo utilizado, com esperança de quadrados médios associados aos diferentes efeitos ${ }^{(1)}$.

\begin{tabular}{|c|c|c|c|c|}
\hline Fonte de variação & GL & QM & $\mathrm{E}(\mathrm{QM})$ & $\mathrm{F}$ \\
\hline Blocos & $b-1$ & $\mathrm{Q}_{1}$ & $\sigma_{b}^{2}+f \sigma_{a}^{2}+f p \sigma_{r}^{2}$ & $\mathrm{Q}_{1} / \mathrm{Q}_{3}$ \\
\hline Procedências & $\mathrm{p}-1$ & $\mathrm{Q}_{2}$ & $\sigma_{\mathrm{b}}^{2}+b \sigma_{\mathrm{f} / \mathrm{p}}^{2}+\mathrm{f} \sigma_{\mathrm{a}}^{2}+\mathrm{fb} \sigma_{\mathrm{p}}^{2}$ & $\mathrm{Q}_{2} / \mathrm{Q}_{3}$ \\
\hline Resíduo (a) & $(b-1)(p-1)$ & $\mathrm{Q}_{3}$ & $\sigma_{\mathrm{b}}^{2}+\mathrm{f} \sigma_{\mathrm{a}}^{2}$ & \\
\hline Progênie/Procedência & $\sum_{i}\left(f_{i}-1\right)$ & $\mathrm{Q}_{4}$ & $\sigma_{\mathrm{b}}^{2}+\mathrm{b} \sigma_{\mathrm{f} / \mathrm{p}}^{2}$ & $\mathrm{Q}_{4} / \mathrm{Q}_{12}$ \\
\hline Progênie/Procedência 1 & $\mathrm{f}_{1}-1$ & $\mathrm{Q}_{5}$ & $\sigma_{\mathrm{b}}^{2}+\mathrm{b} \sigma_{\mathrm{f} / \mathrm{p}_{1}}^{2}$ & $\mathrm{Q}_{5} / \mathrm{Q}_{12}$ \\
\hline Progênie/Procedência 2 & $\mathrm{f}_{2}-1$ & $\mathrm{Q}_{6}$ & $\sigma_{\mathrm{b}}^{2}+\mathrm{b} \sigma_{\mathrm{f} / \mathrm{p}_{2}}^{2}$ & $\mathrm{Q}_{6} / \mathrm{Q}_{12}$ \\
\hline Progênie/Procedência 3 & $f_{3}-1$ & $\mathrm{Q}_{7}$ & $\sigma_{\mathrm{b}}^{2}+\mathrm{b} \sigma_{\mathrm{f} / \mathrm{p}_{3}}^{2}$ & $\mathrm{Q}_{7} / \mathrm{Q}_{12}$ \\
\hline Progênie/Procedência 4 & $\mathrm{f}_{4}-1$ & $\mathrm{Q}_{8}$ & $\sigma_{\mathrm{b}}^{2}+\mathrm{b} \sigma_{\mathrm{f} / \mathrm{p}_{4}}^{2}$ & $\mathrm{Q}_{8} / \mathrm{Q}_{12}$ \\
\hline Progênie/Procedência 5 & $\mathrm{f}_{5}-1$ & $\mathrm{Q}_{9}$ & $\sigma_{\mathrm{b}}^{2}+\mathrm{b} \sigma_{\mathrm{f} / \mathrm{p}_{5}}^{2}$ & $\mathrm{Q}_{9} / \mathrm{Q}_{12}$ \\
\hline Progênie/Procedência 6 & $\mathrm{f}_{6}-1$ & $\mathrm{Q}_{10}$ & $\sigma_{\mathrm{b}}^{2}+\mathrm{b} \sigma_{\mathrm{f} / \mathrm{p}_{6}}^{2}$ & $\mathrm{Q}_{10} / \mathrm{Q}_{12}$ \\
\hline Progênie/Procedência 7 & $\mathrm{f}_{7}-1$ & $\mathrm{Q}_{11}$ & $\sigma_{\mathrm{b}}^{2}+\mathrm{b} \sigma_{\mathrm{f} / \mathrm{p}_{7}}^{2}$ & $\mathrm{Q}_{11} / \mathrm{Q}_{12}$ \\
\hline Erro (b) & $(b-1)\left(\sum_{i} f_{i}-p\right)$ & $\mathrm{Q}_{12}$ & $\sigma_{\mathrm{b}}^{2}$ & \\
\hline Total & $\left(b \sum_{i} f_{i}\right)-1$ & & & \\
\hline
\end{tabular}

(1)p: número de procedências; b: número de blocos; $\mathrm{f}_{\mathrm{i}}$ : número de progênies na procedência $\mathrm{i} ; \sigma_{\mathrm{p}}^{2}$ : variância entre procedências; $\sigma_{\mathrm{f}}^{2}$ p : variância entre progênies dentro de procedências; $\sigma_{\mathrm{r}}^{2}$ : variância entre blocos; $\sigma_{\mathrm{a}}^{2}$ : variância do erro a (variância do erro entre procedências); $\sigma_{\mathrm{b}}^{2}$ : variância do erro b (variância da interação progênies x bloco). 
$\mathrm{I}=\hat{\mathrm{h}}_{\mathrm{d}}^{2}\left(\mathrm{Y}_{\mathrm{ijk}}\right)+\left(\hat{\mathrm{h}}_{\mathrm{p}}^{2}-\hat{\mathrm{h}}_{\mathrm{d}}^{2}\right) \overline{\mathrm{X}}_{\mathrm{ij} .}+\left(\hat{\mathrm{h}}_{\mathrm{b}}^{2}-\hat{\mathrm{h}}_{\mathrm{p}}^{2}\right) \overline{\mathrm{X}}_{\mathrm{j} .}+$

$\left(\hat{\mathrm{h}}_{\mathrm{f}}^{2}-\hat{\mathrm{h}}_{\mathrm{p}}^{2}\right) \overline{\mathrm{X}}_{\mathrm{i} . .}+\left(\hat{\mathrm{h}}_{\mathrm{p}}^{2}-\hat{\mathrm{h}}_{\mathrm{b}}^{2}-\hat{\mathrm{h}}_{\mathrm{f}}^{2}\right) \overline{\mathrm{X}}_{. .}$

$\mathrm{Y}_{\mathrm{ijk}}=$ valor do indivíduo $\mathrm{k}$ na parcela $\mathrm{ij}$;

$\overline{\mathrm{X}}_{\mathrm{i} . .}=$ média da família no ensaio;

$\overline{\mathrm{X}}_{\mathrm{ij} . .}=$ média da família em determinado bloco (média da parcela);

$\overline{\mathrm{X}}_{\mathrm{j} .}=$ média do bloco;

$\hat{\mathrm{h}}_{\mathrm{d}}^{2}=$ herdabilidade no sentido restrito de indivíduo dentro de parcela;

$\hat{\mathrm{h}}_{\mathrm{f}}^{2}=$ herdabilidade no sentido restrito de progênie;

$\hat{\mathrm{h}}_{\mathrm{p}}^{2}=$ herdabilidade no sentido restrito de parcela;

$\hat{\mathrm{h}}_{\mathrm{b}}^{2}=$ herdabilidade no sentido restrito de bloco;

$\overline{\mathrm{X}}_{\ldots}=$ média geral do experimento.

O intervalo de confiança (IC) do ganho genético foi construído através da expressão de Resende et al. (1994a):

$\mathrm{IC}=\mathrm{G}_{\mathrm{S}} \pm \mathrm{t}\left[1 / \mathrm{n}\left[\hat{\sigma}_{\mathrm{A}}^{2}\left(1-\mathrm{r}_{\mathrm{I}, \mathrm{A}}^{2}\right)\right]\right]^{1 / 2}$,

onde $r_{I A}^{2}$ é a acurácia, té o valor tabelado associado à distribuição t de Student. Neste estudo, para 95\% de significância, $\mathrm{t}=1,96$.
Os estimadores da acurácia derivados dos diferentes métodos de seleção estão na Tabela 3, conforme Resende et al. (1994).

\section{Resultados e Discussão}

A análise de variância do volume, forma do fuste e árvores com copa quebrada revelou que existem diferenças significativas de $5 \%$ (teste $\mathrm{F}$ ), entre as médias de todas as progênies, entre procedências e entre progênies dentro de cada procedência, exceto para Las Crucitas, Guaimaca e San Marcos no caso de árvores com copa quebrada (Tabela 4).

Os coeficientes de variação experimental de progênies dentro de procedências para volume e forma do fuste estão entre $10,7 \%$ e $20,9 \%$. Já para árvores com copa quebrada, estes coeficientes estão entre $19,8 \%$ e 57,3\%, valores altos, indicando grande variação dos dados (Tabela 4).

As procedências Las Crucitas, Guaimaca e La Campa apresentaram maior produtividade volumétrica e são originadas de regiões com menores longitudes $\left(86^{\circ} \mathrm{W}\right.$ e $\left.88^{\circ} \mathrm{W}\right)$ e latitudes $\left(13^{\circ} 24^{\prime} \mathrm{N}\right.$ e $\left.14^{\circ} 32^{\prime} \mathrm{N}\right)$, e diferem significativamente, a 5\% (teste de Tukey) das

Tabela 3. Estimadores da acurácia associada a diferentes métodos de seleção ${ }^{(1)}$

\begin{tabular}{|c|c|}
\hline Métodos de seleção & Estimadores \\
\hline & No bloco \\
\hline Individual & $\frac{\hat{\sigma}_{\mathrm{A}}}{\hat{\sigma}_{\mathrm{F}_{\mathrm{ib}}}}$ \\
\hline Combinada & {$\left[\frac{\mathrm{n}-1}{\mathrm{n}}(1-\mathrm{r})^{2} \frac{\hat{\sigma}_{\mathrm{A}}^{2}}{\hat{\sigma}_{\mathrm{d}}^{2}}+\frac{\mathrm{p}-1}{\mathrm{p}}\left(\frac{1+(\mathrm{nb}-1) \mathrm{r}}{\mathrm{nb}}\right)^{2} \frac{\hat{\sigma}_{\mathrm{A}}^{2}}{\hat{\sigma}_{\mathrm{F}}^{2}}\right]^{1 / 2}$} \\
\hline Multiefeito & {$\left[\frac{\mathrm{n}-1}{\mathrm{n}}(1-\mathrm{r})^{2} \frac{\hat{\sigma}_{\mathrm{A}}^{2}}{\hat{\sigma}_{\mathrm{d}}^{2}}+\frac{\mathrm{p}-1}{\mathrm{p}}\left(\frac{1+(\mathrm{nb}-1) \mathrm{r}}{\mathrm{nb}}\right)^{2} \frac{\hat{\sigma}_{\mathrm{A}}^{2}}{\hat{\sigma}_{\bar{F}}^{2}}+\left(\frac{\mathrm{b}-1}{\mathrm{~b}}\right)\left(\frac{\mathrm{p}-1}{\mathrm{p}}\right)\left(\frac{1-\mathrm{r}}{\mathrm{n}}\right)^{2} \hat{\frac{\sigma}{\mathrm{A}}}^{2}\right]^{1 / 2}$} \\
\hline & No experimento \\
\hline Individual & $\frac{\hat{\sigma}_{\mathrm{A}}}{\hat{\sigma}_{\mathrm{F}_{\mathrm{ie}}}}$ \\
\hline Combinada & {$\left[\frac{\mathrm{n}-1}{\mathrm{n}}(1-\mathrm{r})^{2} \frac{\hat{\sigma}_{\mathrm{A}}^{2}}{\hat{\sigma}_{\mathrm{d}}^{2}}+\frac{\mathrm{p}-1}{\mathrm{p}}\left(\frac{1+(\mathrm{nb}-1) \mathrm{r}}{\mathrm{nb}}\right)^{2} \frac{\hat{\sigma}_{\mathrm{A}}^{2}}{\hat{\sigma}_{\overline{\mathrm{F}}}^{2}}\right]^{1 / 2}$} \\
\hline Multiefeito & {$\left[\frac{\mathrm{n}-1}{\mathrm{n}}(1-\mathrm{r})^{2} \frac{\hat{\sigma}_{\mathrm{A}}^{2}}{\hat{\sigma}_{\mathrm{d}}^{2}}+\frac{\mathrm{p}-1}{\mathrm{p}}\left(\frac{1+(\mathrm{nb}-1) \mathrm{r}}{\mathrm{nb}}\right)^{2} \frac{\hat{\sigma}_{\mathrm{A}}^{2}}{\hat{\sigma}_{\overline{\mathrm{F}}}^{2}}+\left(\frac{\mathrm{b}-1}{\mathrm{~b}}\right)\left(\frac{\mathrm{p}-1}{\mathrm{p}}\right)\left(\frac{1-\mathrm{r}}{\mathrm{n}}\right)^{2} \hat{\sigma}_{\mathrm{A}}^{2}+\left(\frac{\mathrm{b}-1}{\mathrm{~b}}\right)\left(\frac{1-\mathrm{r}}{\mathrm{n} p}\right)^{2} \frac{\hat{\sigma}_{\mathrm{A}}^{2}}{\hat{\sigma}_{\overline{\mathrm{B}}}^{2}}\right]^{1 / 2}$} \\
\hline
\end{tabular}

(1) $\hat{\sigma}_{\mathrm{A}}$ : variância genética aditiva; $\hat{\sigma}_{\overline{\mathrm{F}}}$ : variância fenotípica em nível de média de família; $\hat{\sigma}_{\mathrm{d}}$ : variância entre plantas dentro de parcela; $\hat{\sigma}_{\mathrm{F}_{\mathrm{b}}}$ : variância fenotípica em nível de individuo no bloco; $\hat{\sigma}_{\mathrm{F}_{\mathrm{i}}}$ : variância fenotípica em nível de individuo no experimento; $\hat{\sigma}^{2}$ : variância residual em nível de parcela; $\hat{\sigma}_{\overline{\mathrm{B}}}$ : variância fenotípica em nível de médias de bloco (Resende \& Higa, 1994b); r: coeficiente de correlação genética entre indivíduos de uma mesma progênie (1/4 para meios irmãos); n, b e p: números de plantas por parcela, de blocos e progênies, respectivamente. 
procedências San Luís e El Castaño, originadas de regiões de maiores longitudes $\left(89^{\circ} \mathrm{W}\right.$ e $\left.90^{\circ} \mathrm{W}\right)$ e latitudes $\left(14^{\circ} 37^{\prime} \mathrm{N}\right.$ e $\left.15^{\circ} 1^{\prime} \mathrm{N}\right)$ que apresentaram menor produção volumétrica (Tabela 4).

A produção volumétrica da testemunha CO 202 (P. tecunumanii de Moutain Pine Ridge) foi inferior ao de todas as procedências em teste, enquanto o volume médio da testemunha BR 405 ( $P$. oocarpa, Agudos, SP) superou somente a produção volumétrica da procedência El Castaño.

A média geral do caráter forma do fuste $(1,76)$ indicou que as árvores das procedências em teste possuem fustes tendendo à tortuosidade. San Marcos, Guaimaca, Tablazon e Las Crucitas, originadas de regiões com menores longitudes possuem fustes menos tortuosos, diferindo a 5\% (teste de Tukey) das procedências El Castaño, San Luís e La Campa, originadas de regiões de maiores longitudes. Resultados semelhantes foram observados por Massaki (1989).
Em Angatuba, foi observada uma correlação positiva e significativa pelo teste $t$, entre árvores de copa quebrada e a precipitação. A procedência San Marcos, originada da região de menor precipitação, apresentou o menor número de árvores com copa quebrada (Tabela 5).

As procedências El Castaño, La Campa, San Marcos, San Luís e as testemunhas BR 405 ( $P$. oocarpa de Agudos, SP) e CO 202 (P. tecunumanii) apresentaram a maior sobrevivência, e suas médias diferem significativamente a 5\% (teste de Tukey) de Guaimaca, Las Crucitas e Tablazon (Tabela 6).

San Marcos, Las Crucitas, San Luís e a testemunha CO 202 (P. tecunumanii) constituem um grupo de procedências com fibras de maior largura e diâmetro do lúmen, diferindo significativamente a 5\% (teste de Tukey) de La Campa e Tablazon, que apresentaram fibras de menor largura, e de menor diâmetro do lúmen (Tabela 6).

Tabela 4. Análise de variância em nível de progênies e comparação das médias de procedências pelo teste de Tukey para volume, forma do fuste e árvores com copa quebrada de $P$. oocarpa, aos nove anos de idade, em Angatuba, $\mathrm{SP}^{(1)}$.

\begin{tabular}{|c|c|c|c|c|c|c|c|c|c|c|}
\hline \multirow[t]{2}{*}{ Procedências } & \multirow[t]{2}{*}{$\mathrm{N}$} & \multicolumn{3}{|c|}{ Volume (m³/árv.) } & \multicolumn{3}{|c|}{ Forma do fuste } & \multicolumn{3}{|c|}{ Copa quebrada } \\
\hline & & $\overline{\bar{X}}$ & $\mathrm{CV}(\%)$ & $\mathrm{F}$ & $\overline{\bar{X}}$ & $\mathrm{CV}(\%)$ & $\mathrm{F}$ & $\overline{\bar{X}}$ & $\mathrm{CV}(\%)$ & $\mathrm{F}$ \\
\hline Las Crucitas & 8 & $0,310 \mathrm{~A}$ & 15,6 & $3,0 * *$ & $1,78 \mathrm{~B}$ & 17,2 & $2,9 * *$ & $26,6 \mathrm{BC}$ & 45,5 & $0,7^{\mathrm{ns}}$ \\
\hline Guaimaca & 8 & $0,303 \mathrm{AB}$ & 10,7 & $1,4^{*}$ & $1,86 \mathrm{AB}$ & 20,0 & $5,7 * *$ & $24,3 \mathrm{D}$ & 49,4 & $0,8^{\text {ns }}$ \\
\hline San Marcos & 8 & $0,299 \mathrm{BC}$ & 14,2 & $1,5^{* *}$ & $1,89 \mathrm{~A}$ & 17,8 & $5,9 * *$ & $22,1 \mathrm{E}$ & 45,1 & $1,2^{\mathrm{ns}}$ \\
\hline La Campa & 8 & $0,296 \mathrm{C}$ & 12,6 & $2,4 * *$ & $1,65 \mathrm{C}$ & 20,9 & $12,2 * *$ & $27,9 \mathrm{~B}$ & 56,6 & $2,6^{* * *}$ \\
\hline Tablazon & 6 & $0,293 \mathrm{CD}$ & 16,4 & $6,7 * *$ & $1,79 \mathrm{~B}$ & 18,8 & $6,5^{* *}$ & $25,6 \mathrm{CD}$ & 19,8 & $1,4^{*}$ \\
\hline San Luis & 8 & $0,292 \mathrm{D}$ & 16,5 & $3,6^{* *}$ & $1,67 \mathrm{C}$ & 18,4 & $4,8 * *$ & $25,6 \mathrm{CD}$ & 46,3 & $4,0 * *$ \\
\hline El Castaño & 8 & $0,282 \mathrm{E}$ & 15,3 & $2,7 * *$ & $1,69 \mathrm{C}$ & 19,8 & $9,2 * *$ & $26,8 \mathrm{BC}$ & 57,3 & $2,2 * *$ \\
\hline TEST $1^{(2)}$ & & $0,258 \mathrm{~F}$ & & & $1,39 \mathrm{D}$ & & & $31,7 \mathrm{~A}$ & & \\
\hline TEST $2^{(3)}$ & & $0,288 \mathrm{E}$ & & & $1,69 \mathrm{C}$ & & & $26,3 \mathrm{BC}$ & & \\
\hline Média Geral & & $0,296^{(4)}$ & & & $1,76^{(4)}$ & & & $25,5^{(4)}$ & & \\
\hline F prog/proc (em conjunto) & & & & $3,1 * *$ & & & $6,8 * *$ & & & $1,9 * *$ \\
\hline F proc (entre procedências) & & & & $4,3^{* *}$ & & & $4,2 * *$ & & & \\
\hline
\end{tabular}

(1) Médias seguidas pelas mesmas letras não diferem significativamente a 5\% pelo teste de Tukey; N: número de progênies por procedência; CV: coeficiente de variação em nível de progênie; $\overline{\mathrm{X}}$ : média das procedências. ${ }^{(2)}$ TEST 1: P. tecunumanii (Mountain Pine Ridge). ${ }^{(3)}$ TEST 2: P. oocarpa (Agudos, SP).

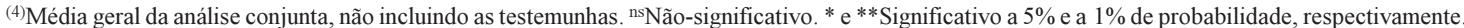

Tabela 5. Coeficiente de correlação de Spearman entre características de crescimento de $P$. oocarpa em Angatuba, SP, com os dados ambientais da origem das sementes.

\begin{tabular}{|c|c|c|c|c|c|c|c|c|}
\hline \multirow[t]{2}{*}{ Características } & \multicolumn{2}{|c|}{ Latitude } & \multicolumn{2}{|c|}{ Longitude } & \multicolumn{2}{|c|}{ Altitude } & \multicolumn{2}{|c|}{ Precipitação } \\
\hline & $\mathrm{r}_{1}^{2}$ & $\mathrm{t}$ & $\mathrm{r}_{1}^{2}$ & $\mathrm{t}$ & $\mathrm{r}_{1}^{2}$ & $\mathrm{t}$ & $\mathrm{r}_{1}^{2}$ & $\mathrm{t}$ \\
\hline Volume & $-0,678$ & $2,06 *$ & $-0,964$ & $8,10 *$ & $-0,214$ & $0,49^{\mathrm{ns}}$ & $-0,250$ & $0,58^{\mathrm{ns}}$ \\
\hline Forma do fuste & $-0,535$ & $1,41^{\mathrm{ns}}$ & $-0,818$ & $3,12 *$ & $-0,392$ & $0,95^{\mathrm{ns}}$ & $-0,464$ & $1,20^{\mathrm{ns}}$ \\
\hline Copa quebrada & 0,321 & $0,75^{\mathrm{ns}}$ & 0,392 & $0,95^{\mathrm{ns}}$ & 0,642 & $1,87^{\mathrm{ns}}$ & 0,750 & $2,53 *$ \\
\hline Densidade básica & $-0,142$ & $0,32^{\text {ns }}$ & $-0,607$ & $1,70^{\mathrm{ns}}$ & $-0,178$ & $0,40^{\mathrm{ns}}$ & $-0,178$ & $0,40^{\mathrm{ns}}$ \\
\hline
\end{tabular}

ns Não-significativo. ${ }^{*}$ Significativo a $10 \%$ de probabilidade pelo teste $\mathrm{t}$. 
A densidade básica média da madeira de P. oocarpa em Angatuba, aos nove anos de idade foi de $0,410 \mathrm{~g} / \mathrm{cm}^{3}$, valor aproximado ao recomendado por Arbuthnot (1991) quanto à produção de papel kraft, que é de $0,460 \mathrm{~g} / \mathrm{cm}^{3}$. Valores semelhantes foram observados por Amaral et al. (1977) e Barrichelo (1984). A densidade básica da madeira e os índices entre as dimensões das fibras podem ser consideradas como características de grande importância quanto às propriedades físico-mecânicas do papel. Com base nestas características, pode-se concluir que a madeira de $P$. oocarpa é indicada para a produção de celulose e papel kraft.

Os coeficientes de herdabilidade no sentido restrito de indivíduos foram menores que os estimados em nível de médias de famílias dos caracteres volume e forma do fuste (Tabela 7), revelando que a seleção destes caracteres pode ser mais efetiva em nível de médias de progênies que dentro de progênies. A baixa magnitude desses coeficientes de herdabilidade indica que os efeitos ambientais foram preponderantes na manifestação destas características em todas as procedências.

Para o caráter densidade básica da madeira, os coeficientes de herdabilidade restrito de famílias e de indivíduos foram de elevada magnitude nas procedências El Castaño, Guaimaca e Las Crucitas, o que indica que a seleção poderia ser conduzida em nível de indivíduos. Os caracteres que apresentaram os maiores coeficientes de herdabilidade restrito, em ordem decrescente, são: forma do fuste, densidade básica da madeira, e volume.

Estimativas de herdabilidade associadas a altos desvios-padrões tornam duvidosas as previsões de ganho genético esperado com a seleção (Souza et al., 1992). Este fato pode ser observado nas procedências La Campa, San Luís e Tablazon, onde o coeficiente de herdabilidade da densidade básica da madeira das médias de famílias possui magnitude média e está associado a elevado desvio-padrão (Tabela 7).

$\mathrm{O}$ experimento em estudo foi instalado com seis plantas por parcela e oito progênies por procedência, porém com sobrevivência em torno de $72,4 \%$, o que resulta em quatro indivíduos por parcela. Esta diminuição do número de indivíduos por parcela implica numa redução da variância genética aditiva no cálculo da herdabilidade em nível de média de parcela de $17,4 \%$, justificando a inclusão do efeito de parcela nos processos de seleção (Resende \& Fernandes, 1999).

As estimativas dos coeficientes de correlação genética aditiva de plantas $\left(\mathrm{r}_{\mathrm{A}}\right)$ foram altas e positivas entre os caracteres altura, DAP e volume (Tabela 8). O DAP mostrou-se altamente correlacionado com o

Tabela 6. Análise de variância de progênies e procedências para densidade básica da madeira e sobrevivência de procedências para comprimento de fibra (C), largura de fibra (L), diâmetro do lúmen (DL), espessura da parede das fibras (EP) e comparação das médias de procedências pelo teste de Tukey de $P$. oocarpa, aos nove anos de idade, em Angatuba, $\mathrm{SP}^{(1)}$

\begin{tabular}{|c|c|c|c|c|c|c|c|c|c|c|}
\hline \multirow[t]{2}{*}{ Procedências } & \multicolumn{3}{|c|}{$\begin{array}{c}\text { Densidade básica } \\
\left(\mathrm{g} / \mathrm{cm}^{3}\right)\end{array}$} & \multicolumn{3}{|c|}{$\begin{array}{c}\text { Sobrevivência } \\
(\%)\end{array}$} & \multirow[t]{2}{*}{$\begin{array}{c}\mathrm{C} \\
(\mathrm{mm})\end{array}$} & \multirow[t]{2}{*}{$\begin{array}{c}\mathrm{L} \\
(\mu)\end{array}$} & \multirow[t]{2}{*}{$\begin{array}{l}\text { DL } \\
(\mu)\end{array}$} & \multirow[t]{2}{*}{$\begin{array}{l}\mathrm{EP} \\
(\mu)\end{array}$} \\
\hline & $\bar{X}$ & $\mathrm{CV}(\%)$ & $\mathrm{F}$ & $\overline{\mathrm{X}}$ & $\mathrm{CV}(\%)$ & $\mathrm{F}$ & & & & \\
\hline La Campa & $0,412 \mathrm{~A}$ & 10,9 & $1,6 * *$ & $76,0 \mathrm{BC}$ & 19,0 & $0,6^{\mathrm{ns}}$ & $3,2 \mathrm{~A}$ & $51,9 \mathrm{CD}$ & $35,0 \mathrm{DE}$ & $8,4 \mathrm{~A}$ \\
\hline El Castaño & $0,409 \mathrm{~A}$ & 6,1 & $2,4 * *$ & $78,3 \mathrm{AB}$ & 17,7 & $2,6^{* *}$ & $3,1 \mathrm{~A}$ & $53,0 \mathrm{BC}$ & $36,7 \mathrm{CD}$ & $8,1 \mathrm{~A}$ \\
\hline Guaimaca & $0,414 \mathrm{~A}$ & 7,7 & $4,0 * *$ & $64,0 \mathrm{E}$ & 27,4 & $0,4^{\mathrm{ns}}$ & $3,3 \mathrm{~A}$ & $53,0 \mathrm{AB}$ & $36,4 \mathrm{CD}$ & $8,3 \mathrm{~A}$ \\
\hline San Marcos & $0,409 \mathrm{~A}$ & 5,8 & $0,9^{\mathrm{ns}}$ & $75,8 \mathrm{BC}$ & 19,9 & $0,7^{\mathrm{ns}}$ & $3,3 \mathrm{~A}$ & $53,5 \mathrm{AB}$ & $37,0 \mathrm{BC}$ & $8,2 \mathrm{~A}$ \\
\hline Las Crucitas & $0,415 \mathrm{~A}$ & 6,1 & $2,4 * *$ & $70,3 \mathrm{D}$ & 23,5 & $1,6^{* *}$ & $3,3 \mathrm{~A}$ & $54,6 \mathrm{~A}$ & $38,3 \mathrm{AB}$ & $8,2 \mathrm{~A}$ \\
\hline San Luís & $0,411 \mathrm{~A}$ & 6,5 & $1,1^{\mathrm{ns}}$ & $74,6 \mathrm{C}$ & 19,3 & $1,5^{*}$ & $3,1 \mathrm{~A}$ & $53,5 \mathrm{AB}$ & $37,9 \mathrm{AB}$ & $7,8 \mathrm{~A}$ \\
\hline Tablazon & $0,400 \mathrm{~B}$ & 6,2 & $2,5 * *$ & $67,7 \mathrm{D}$ & 24,0 & $1,3^{\mathrm{ns}}$ & $3,2 \mathrm{~A}$ & $50,7 \mathrm{D}$ & $33,9 \mathrm{E}$ & $8,4 \mathrm{~A}$ \\
\hline TEST $1^{(2)}$ & $0,414 \mathrm{~A}$ & & & $79,3 \mathrm{~A}$ & & & $3,2 \mathrm{~A}$ & $54,6 \mathrm{~A}$ & $39,0 \mathrm{~A}$ & $7,8 \mathrm{~A}$ \\
\hline TEST $2^{(3)}$ & $0,408 \mathrm{~A}$ & & & $78,0 \mathrm{AB}$ & & & & & & \\
\hline Média geral $^{(4)}$ & 0,410 & & & 72,4 & & & 3,2 & 53,1 & 36,8 & 8,2 \\
\hline Fprog/proc (em conjunto) & & & $0,7^{\mathrm{ns}}$ & & & $1,2^{\mathrm{ns}}$ & & & & \\
\hline Fproc (entre procedências) & & & $0,9^{\mathrm{ns}}$ & & & $7,3^{* *}$ & $0,6^{\mathrm{ns}}$ & $2,4 * *$ & $3,3 * *$ & $0,9^{\mathrm{ns}}$ \\
\hline
\end{tabular}


volume, e os desvios-padrões destas correlações foram de baixa magnitude, indicando, assim, que a opção da seleção baseada no DAP deverá refletir-se em estimativas de ganhos genéticos expressivos em volume com boa precisão. Desta forma, a primeira etapa da seleção visando elevar a produção

Tabela 7. Estimativas de herdabilidade no sentido restrito de indivíduos na parcela $\left(\mathrm{h}^{2}{ }_{\mathrm{ip}}\right)$, de progênies $\left(\mathrm{h}_{\mathrm{f}}^{2}\right)$, de parcela $\left(\mathrm{h}_{\mathrm{p}}^{2}\right)$, de indivíduo no bloco $\left(\mathrm{h}^{2}{ }_{\mathrm{ib}}\right)$ e de indivíduo no experimento $\left(\mathrm{h}^{2}{ }_{\mathrm{ie}}\right)$ para as características de crescimento e da densidade básica da madeira de P. oocarpa, aos nove anos de idade, em Angatuba, SP.

\begin{tabular}{|c|c|c|c|}
\hline Herdabilidade & Volume & Forma do fuste & Densidade básica \\
\hline \multicolumn{4}{|c|}{ La Campa (Honduras) } \\
\hline$h^{2}{ }_{i p}$ & 0,110 & 0,940 & 0,148 \\
\hline$h_{f}^{2(1)}$ & $0,734( \pm 0,126)$ & $0,952( \pm 0,050)$ & $0,476( \pm 0,343)$ \\
\hline$h_{p}^{2}$ & 0,144 & 0,546 & 0,154 \\
\hline $\mathrm{h}^{2_{\mathrm{ib}}^{\mathrm{F}}}$ & 0,149 & 0,864 & 0,190 \\
\hline $\mathrm{h}_{\mathrm{ie}}^{2}$ & 0,147 & 0,854 & 0,190 \\
\hline \multicolumn{4}{|c|}{ El Castaño (Guatemala) } \\
\hline $\mathrm{h}_{\mathrm{ip}}^{2}$ & 0,114 & 0,575 & 0,716 \\
\hline $\mathrm{h}_{\mathrm{f}}^{2(1)}$ & $0,641( \pm 0,179)$ & $0,928( \pm 0,058)$ & $0,855( \pm 0,175)$ \\
\hline$h_{p}^{2}$ & 0,096 & 0,434 & 0,542 \\
\hline $\mathrm{h}^{2}{ }_{\mathrm{ib}}^{\mathrm{P}}$ & 0,141 & 0,612 & 0,709 \\
\hline$\underline{\mathrm{h}_{\mathrm{ie}}^{2}}$ & 0,142 & 0,600 & 0,719 \\
\hline \multicolumn{4}{|c|}{ Guaimaca (Guatemala) } \\
\hline$h_{\text {ip }}^{2}$ & 0,104 & 0,418 & 0,479 \\
\hline $\mathrm{h}_{\mathrm{f}}^{2(1)}$ & $0,669( \pm 0,237)$ & $0,839( \pm 0,10)$ & $0,941( \pm 0,290)$ \\
\hline $\mathrm{h}_{\mathrm{p}}^{2}$ & 0,138 & 0,293 & 0,764 \\
\hline $\mathrm{h}^{2}{ }_{\mathrm{ib}}$ & 0,143 & 0,443 & 0,617 \\
\hline $\mathrm{h}_{\mathrm{ie}}^{2}$ & 0,144 & 0,436 & 0,625 \\
\hline \multicolumn{4}{|c|}{ San Marcos (Honduras) } \\
\hline$h^{2}{ }_{i p}$ & 0,040 & 0,346 & (2) \\
\hline $\mathrm{h}_{\mathrm{f}}^{2(1)}$ & $0,486( \pm 0,275)$ & $0,846( \pm 0,109)$ & (2) \\
\hline$h_{p}^{2}$ & 0,054 & 0,251 & (2) \\
\hline $\mathrm{h}^{2}{ }_{\mathrm{ib}}$ & 0,056 & 0,388 & (2) \\
\hline $\mathrm{h}_{\mathrm{ie}}^{2}$ & 0,056 & 0,361 & (2) \\
\hline \multicolumn{4}{|c|}{ Las Crucitas (Honduras) } \\
\hline$h_{\text {ip }}^{2}$ & 0,085 & 0,086 & 0,315 \\
\hline$h_{f}^{2(1)}$ & $0,632( \pm 0,207)$ & $0,639( \pm 0,194)$ & $0,731( \pm 0,230)$ \\
\hline$h_{p}^{2}$ & 0,102 & 0,104 & 0,352 \\
\hline $\mathrm{h}_{\mathrm{ib}}^{2}$ & 0,114 & 0,115 & 0,393 \\
\hline $\mathrm{h}_{\mathrm{ie}}^{2}$ & 0,115 & 0,113 & 0,382 \\
\hline \multicolumn{4}{|c|}{ San Luís (Guatemala) } \\
\hline$h_{\text {ip }}^{2}$ & 0,153 & 0,302 & 0,039 \\
\hline$h_{f}^{2(1)}$ & $0,707( \pm 0,171)$ & $0,855( \pm 0,090)$ & $0,155( \pm 0,486)$ \\
\hline$h_{p}^{2}$ & 0,129 & 0,269 & 0,035 \\
\hline $\mathrm{h}^{2}{ }_{\mathrm{ib}}$ & 0,188 & 0,357 & 0,050 \\
\hline $\mathrm{h}_{\mathrm{ie}}^{2}$ & 0,189 & 0,351 & 0,049 \\
\hline \multicolumn{4}{|c|}{ Tablazon (Honduras) } \\
\hline$h^{2}{ }_{i p}$ & 0,327 & 0,490 & 0,048 \\
\hline $\mathrm{h}_{\mathrm{f}}^{2(1)}$ & $0,867( \pm 0,111)$ & $0,887( \pm 0,101)$ & $0,556( \pm 0,624)$ \\
\hline$h_{p}^{2}$ & 0,314 & 0,355 & 0,200 \\
\hline $\mathrm{h}^{2}{ }_{\mathrm{ib}}$ & 0,390 & 0,523 & 0,084 \\
\hline $\mathrm{h}_{\mathrm{ie}}^{2}$ & 0,399 & 0,526 & 0,083 \\
\hline
\end{tabular}

Tabela 8. Estimativa das correlações genéticas aditivas de plantas $\left(\mathrm{r}_{\mathrm{A}}\right.$ e seus desvios-padrões $\left[\mathrm{S}\left(\mathrm{r}_{\mathrm{Axy}}\right)\right]$ e fenotípicas $\left(\mathrm{r}_{\mathrm{F}}\right)$ entre os pares de caracteres de crescimento de P. oocarpa, aos nove anos de idade, em Angatuba, SP.

\begin{tabular}{lccc}
\hline Características & $\mathrm{r}_{\mathrm{F}}{ }^{(1)}$ & $\mathrm{r}_{\mathrm{A}}{ }^{(2)}$ & $\mathrm{S}\left(\mathrm{r}_{\mathrm{Axy}}\right)$ \\
\hline & La Campa (Honduras) & \\
Altura/DAP & 0,789 & 0,758 & 0,266 \\
Altura/volume & 0,894 & 0,802 & 0,218 \\
Altura/densidade & 0 & $-0,082$ & 0,977 \\
DAP/volume & 0,914 & 0,987 & 0,012 \\
DAP/densidade & $-0,710$ & $-0,475$ & 0,646 \\
Volume/densidade & $-0,630$ & $-0,359$ & 0,709
\end{tabular}

\begin{tabular}{lccc}
\hline & El Castaño (Guatemala) & \\
Altura/DAP & 0,848 & 0,701 & 0,481 \\
Altura/volume & 0,868 & 0,784 & 0,330 \\
Altura/densidade & 0 & 0,275 & 0,682 \\
DAP/volume & 0,923 & 0,977 & 0,033 \\
DAP/densidade & 0 & $-0,256$ & 0,587 \\
Volume/densidade & 0 & $-0,200$ & 0,544
\end{tabular}

\begin{tabular}{lccl} 
Volume/densidade & 0 & $-0,200$ & 0,544 \\
\hline \multicolumn{4}{c}{ Guaimaca (Honduras) } \\
Altura/DAP & 0,943 & 0,489 & \multicolumn{1}{l}{$(3)$} \\
Altura/volume & 0,715 & 0,795 & 0,213 \\
Altura/densidade & 0 & $-0,290$ & 0,508 \\
DAP/volume & 0,998 & 0,916 & $(3)$ \\
DAP/densidade & 0 & $-0,371$ & $(3)$ \\
Volume/densidade & 0 & $-0,329$ & 0,570 \\
\hline \multicolumn{4}{c}{ San Marcos (Honduras) } \\
Altura/DAP & 0,848 & 0,576 & 0,672 \\
Altura/volume & 0,890 & 0,659 & 0,645 \\
Altura/densidade & 0 & $-0,600$ & $(3)$ \\
DAP/volume & 0,987 & 0,990 & 0,019 \\
DAP/densidade & 0 & $-0,467$ & $(3)$ \\
Volume/densidade & 0 & $-0,524$ & $(3)$
\end{tabular}

\begin{tabular}{lccc}
\hline & \multicolumn{3}{c}{ Las Crucitas (Honduras) } \\
Altura/DAP & 0,991 & 0,856 & 0,209 \\
Altura/volume & 0,990 & 0,921 & 0,114 \\
Altura/densidade & 0 & $-0,442$ & 0,580 \\
DAP/volume & 0,990 & 0,972 & 0,041 \\
DAP/densidade & 0 & 0,268 & 0,660 \\
Volume/densidade & 0 & 0,086 & 0,680 \\
\hline \multicolumn{4}{c}{ San Luís (Guatemala) } \\
Altura/DAP & 0,785 & 0,535 & 0,480 \\
Altura/volume & 0,915 & 0,760 & 0,266 \\
Altura/densidade & 0 & 0,498 & 0,972 \\
DAP/volume & 0,977 & 0,956 & 0,044 \\
DAP/densidade & 0,369 & 0,259 & 1,680 \\
Volume/densidade & 0,360 & 0,323 & 1,540 \\
\hline & Tablazon (Honduras) & \\
Altura/DAP & 0,941 & 0,428 & 0,340 \\
Altura/volume & 0,913 & 0,526 & 0,279 \\
Altura/densidade & 0 & 0,662 & 0,764 \\
DAP/volume & 0,999 & 0,990 & 0,005 \\
DAP/densidade & 0 & $-0,316$ & 0,782 \\
Volume/densidade & 0 & $-0,029$ & 0,882 \\
\hline
\end{tabular}

${ }^{(1)} \mathrm{r}_{\mathrm{A}}$ : coeficiente de correlação genética aditiva. ${ }^{(2)} \mathrm{r}_{\mathrm{F}}$ : coeficiente de correlação fenotípica. ${ }^{(3)}$ Não foi possível estimar o desvio-padrão, pelo fato de o valor de F ser inferior a 1 . 
volumétrica deverá ser feita com base no DAP (Tabela 8 ).

A correlação fenotípica é uma combinação da correlação genética e de ambiente, e está também relacionada à herdabilidade dos dois caracteres correlacionados (Matziris \& Zobel, 1973; Kageyama, 1980; Vencovsky \& Barriga, 1992). Deste modo, as altas correlações fenotípicas encontradas entre altura, DAP e volume deveram-se tanto aos efeitos ambientais como aos genéticos (Tabela 8). Deve-se considerar que as correlações obtidas foram em níveis diferentes: uma, em nível de plantas, e outra, em nível de médias de famílias, não possibilitando, portanto, comparações diretas.

A maioria dos testes de progênies delineados em blocos de famílias compactas vem utilizando várias procedências com um número de progênies muito pequeno que sob seleção poderia resultar num esquema inadequado para a produção de sementes (Wrigth, 1976).

Neste trabalho, optou-se por ignorar as diferenças entre procedências e selecionar o mesmo número de indivíduos em cada procedência, com base nos maiores ganhos genéticos em volume através de seleção no DAP. O fato de utilização de todas as procedências é decorrente da necessidade do resgate do material genético das populações em estudo e, conseqüentemente, da ampliação da base genética e da racionalização da disposição dos indivíduos na área do pomar de sementes por mudas após o desbaste.

As acurácias e os intervalos de confiança foram os parâmetros usados para a determinação do método de seleção, que possibilitou as maiores estimativas de ganho genético em volume via seleção em DAP (Tabela 9).

Para a instalação do pomar de sementes por mudas a partir do teste de procedência e progênies de P. oocarpa, localizado em Angatuba, SP, foram selecionados 27 indivíduos por procedência. $\mathrm{O}$ número de indivíduos selecionados por procedência correspondeu a uma intensidade de seleção entre $8,33 \%$ (Tablazon) e $6,25 \%$ (demais procedências), o que deixou o pomar com 189 indivíduos distribuídos de maneira equilibrada, numa área de 3,18 ha.

A seleção destes indivíduos proporcionou estimativas do ganho genético indireto em volume, através da seleção individual de $10,46 \%$, na seleção combinada de $14,08 \%$ e no índice multiefeito de $14,46 \%$. Elevou-se, assim, a nova média das populações em apenas um ciclo de seleção de 0,2964 m²árv. para $0,3272 \mathrm{~m}^{3} /$ árv. (seleção individual), para $0,3388 \mathrm{~m}^{3}$ /árv. (seleção combinada) e para $0,3418 \mathrm{~m}^{3} /$ árv. (índice multiefeito) (Tabela 10).

As acurácias e os limites inferiores dos intervalos de segurança dos ganhos genéticos indiretos do volume referente ao método de seleção denominado índice multiefeito foram, no mínimo, iguais às obtidas para a seleção combinada no bloco e sempre superiores às obtidas para seleção individual. Logo, é aconselhável optar pela utilização do índice multiefeito, pois sua adoção não implica custos adicionais ao processo seletivo, e maximizou o ganho genético indireto em volume (Tabela 9).

Este fato é esperado, já que os métodos de seleção entre e dentro de progênies (Kageyama \& Vencovsky, 1983) e seleção combinada (Resende \& Higa, 1994b), normalmente utilizam, para a seleção, apenas duas fontes de informações: o desvio do valor individual em relação à média de família no bloco, e da média da família em relação à média geral do teste de progênie. Porém, em esquemas de melhoramento sem o emprego de sementes remanescentes e com baixo número de plantas por parcela, usando-se apenas estas duas fontes de informações, frações da variância genética aditiva não são consideradas na seleção, ficando retidas nos efeitos de parcela (Resende \& Higa, 1994b).

A seleção em nível de experimento objetiva a instalação de pomar de sementes clonais (PSC). O número de indivíduos selecionados corresponde a uma intensidade de seleção entre 1,23\% (Tablazon) e $0,93 \%$ (demais procedências). Foram selecionados quatro indivíduos por procedência, que apresentaram os maiores ganhos genéticos em DAP, totalizando 28 indivíduos no pomar de sementes clonais.

Na implantação do PSC, a seleção individual proporcionou estimativas de ganhos genéticos indiretos em volume de $16,6 \%$, a seleção combinada de $20 \%$ e o índice multiefeito de $20,6 \%$. Elevando a nova média da população após um ciclo de seleção de 0,2964 m³/árv. para 0,3457 m³/árv. (seleção individual), para $0,3550 \mathrm{~m}^{3} /$ árv. (seleção combinada) e para $0,3564 \mathrm{~m}^{3} /$ árv. (índice multiefeito) (Tabela 10). 
Tabela 9. Acurácias associadas às unidades de seleção univariada e porcentagem do ganho genético indireto em volume (GS) através da seleção com base no DAP em nível de bloco e experimento.

\begin{tabular}{|c|c|c|c|c|}
\hline \multirow[t]{2}{*}{ Método de seleção } & \multicolumn{2}{|c|}{ Volume } & \multirow{2}{*}{$\begin{array}{l}\text { Intervalo de confiança } \\
\text { do GS em volume }\end{array}$} & \multirow{2}{*}{$\begin{array}{l}\text { Nova média da população } \\
\text { após a seleção ( } \mathrm{m}^{3} / \mathrm{arv} \text {.) }\end{array}$} \\
\hline & Acurácia & GS $(\%)$ & & \\
\hline \multicolumn{5}{|c|}{ La Campa (Honduras) } \\
\hline & & & No bloco ${ }^{(1)}$ & \\
\hline Individual & 0,386 & 13,62 & $10,4-16,8$ & 0,331 \\
\hline Combinada & 0,488 & 18,26 & $15,3-21,3$ & 0,347 \\
\hline \multirow[t]{2}{*}{ Multiefeito } & 0,504 & 18,96 & $15,9-21,9$ & 0,349 \\
\hline & & & No experimento ${ }^{(2)}$ & \\
\hline Individual & 0,384 & 24,81 & $16,4-33,2$ & 0,364 \\
\hline Combinada & 0,488 & 27,17 & $19,3-35,0$ & 0,373 \\
\hline Multiefeito & 0,505 & 28,11 & $20,3-35,9$ & 0,373 \\
\hline \multicolumn{5}{|c|}{ El Castaño (Guatemala) } \\
\hline \multicolumn{5}{|c|}{ No bloco ${ }^{(1)}$} \\
\hline Individual & 0,414 & 9,49 & $6,2-12,8$ & 0,308 \\
\hline Combinada & 0,493 & 11,68 & $8,6-14,8$ & 0,315 \\
\hline Multiefeito & 0,506 & 12,39 & $9,3-15,5$ & 0,317 \\
\hline & & & No experimento ${ }^{(2)}$ & \\
\hline Individual & 0,416 & 12,66 & $4,1-21,2$ & 0,317 \\
\hline Combinada & 0,493 & 15,33 & $7,2-23,4$ & 0,345 \\
\hline Multiefeito & 0,509 & 15,42 & $7,4-23,4$ & 0,325 \\
\hline \multicolumn{5}{|c|}{ Guaimaca (Honduras) } \\
\hline & & & No bloco ${ }^{(1)}$ & \\
\hline Individual & 0,379 & 4,50 & $2,1-6,9$ & 0,317 \\
\hline Combinada & 0,466 & 9,62 & $7,7-11,5$ & 0,323 \\
\hline Multiefeito & 0,488 & 9,62 & $7,7-11,6$ & 0,333 \\
\hline & & & No experimento ${ }^{(2)}$ & \\
\hline Individual & 0,379 & 6,84 & $1,6-12,0$ & 0,324 \\
\hline Combinada & 0,466 & 12,00 & $7,0-17,0$ & 0,340 \\
\hline Multiefeito & 0,491 & 12,00 & $7,2-16,8$ & 0,340 \\
\hline \multicolumn{5}{|c|}{ San Marcos (Honduras) } \\
\hline & & & No bloco ${ }^{(1)}$ & \\
\hline Individual & 0,212 & 2,72 & $0,7-4,7$ & 0,307 \\
\hline Combinada & 0,335 & 5,11 & $3,2-7,0$ & 0,314 \\
\hline Multiefeito & 0,342 & 5,11 & $3,2-7,0$ & 0,314 \\
\hline & & & No experimento ${ }^{(2)}$ & \\
\hline Individual & 0,213 & 3,87 & - & 0,310 \\
\hline Combinada & 0,335 & 6,32 & $1,3-11,3$ & 0,318 \\
\hline Multiefeito & 0,343 & 6,32 & $1,3-11,1$ & 0,318 \\
\hline \multicolumn{5}{|c|}{ Las Crucitas (Honduras) } \\
\hline & & & No bloco ${ }^{(1)}$ & \\
\hline Individual & 0,338 & 7,49 & $4,0-10,9$ & 0,328 \\
\hline Combinada & 0,445 & 9,28 & $6,0-12,6$ & 0,331 \\
\hline Multiefeito & 0,459 & 9,79 & $6,5-13,0$ & 0,334 \\
\hline & & & No experimento ${ }^{(2)}$ & \\
\hline Individual & 0,339 & 7,97 & - & 0,337 \\
\hline Combinada & 0,445 & 11,14 & $2,6-19,7$ & 0,337 \\
\hline Multiefeito & 0,463 & 12,13 & $3,7-20,6$ & 0,339 \\
\hline
\end{tabular}


Tabela 9. Continuação.

\begin{tabular}{|c|c|c|c|c|}
\hline \multirow[t]{2}{*}{ Método de seleção } & \multicolumn{2}{|c|}{ Volume } & \multirow{2}{*}{$\begin{array}{l}\text { Intervalo de confiança } \\
\text { do GS em volume }\end{array}$} & \multirow{2}{*}{$\begin{array}{l}\text { Nova média da população } \\
\text { após a seleção ( } \mathrm{m}^{3} / \text { arv.) }\end{array}$} \\
\hline & Acurácia & GS (\%) & & \\
\hline \multicolumn{5}{|c|}{ San Luís (Guatemala) } \\
\hline \multicolumn{5}{|c|}{ No bloco ${ }^{(1)}$} \\
\hline Individual & 0,436 & 12,21 & $8,4-16,0$ & 0,327 \\
\hline Combinada & 0,508 & 17,64 & $14,0-21,2$ & 0,343 \\
\hline Multiefeito & 0,523 & 17,81 & $14,3-21,3$ & 0,344 \\
\hline & & & No experimento ${ }^{(2)}$ & \\
\hline Individual & 0,438 & 18,75 & $8,9-28,5$ & 0,346 \\
\hline Combinada & 0,508 & 23,50 & $14,1-32,9$ & 0,360 \\
\hline Multiefeito & 0,526 & 25,07 & $15,7-34,3$ & 0,365 \\
\hline \multicolumn{5}{|c|}{ Tablazon (Honduras) } \\
\hline \multicolumn{5}{|c|}{$\begin{array}{l}\text { Iabrazon (Honquras) } \\
\text { No bloco }\end{array}$} \\
\hline Individual & 0,624 & 23,23 & $18,3-28,1$ & 0,369 \\
\hline Combinada & 0,620 & 27,00 & $22,2-31,8$ & 0,380 \\
\hline Multiefeito & 0,651 & 27,60 & $22,9-32,3$ & 0,382 \\
\hline & & & No experimento ${ }^{(2)}$ & \\
\hline Individual & 0,631 & 41,35 & $28,6-54,0$ & 0,420 \\
\hline Combinada & 0,620 & 45,13 & $32,3-57,9$ & 0,432 \\
\hline Multiefeito & 0,666 & 45,59 & $33,2-58,0$ & 0,436 \\
\hline
\end{tabular}

${ }^{(1)}$ Seleção no bloco: pomar de sementes por mudas. ${ }^{(2)}$ Seleção no experimento: pomar de sementes clonais.

Tabela 10. Acurácias e ganhos genéticos (GS) e a nova média das populações após um ciclo de seleção de $P$. oocarpa, aos nove anos de idade, em Angatuba, SP.

\begin{tabular}{lccc}
\hline Métodos de seleção & \multicolumn{3}{c}{ Volume } \\
\cline { 2 - 4 } & Acurácias & Ganho genético (GS) & $\begin{array}{c}\text { Nova média da população } \\
\left(\mathrm{m}^{3} / \text { arv. }\right)\end{array}$ \\
\hline Individual & 0,398 & No bloco (PSM) & 0,3272 \\
Combinada & 0,479 & 10,46 & 0,3388 \\
Multiefeito & 0,496 & 14,08 & 0,3418 \\
\hline & & 14,46 & 0,3457 \\
Individual & 0,400 & No experimento (PSC) & 0,3550 \\
Combinada & 0,479 & 16,60 & 0,3564 \\
Multiefeito & 0,500 & 20,00 & 20,60 \\
\hline
\end{tabular}

${ }^{(1)}$ Seleção no bloco: pomar de sementes por mudas. ${ }^{(2)}$ Seleção no experimento: pomar de sementes clonais.

A seleção índice multiefeito apresentou acurácias e ganhos genéticos indiretos em volume maiores ou iguais a seleção combinada, e esta, maior que a seleção individual na implantação do pomar de sementes clonais. Resultado similar foi observado para o pomar de sementes por mudas (Tabela 10).

Com base nos ganhos genéticos e nas acurácias, pode-se inferir que a seleção índice multiefeito maximizou os ganhos genéticos indiretos em volume tanto na implantação do pomar de sementes por mudas como no pomar de sementes clonais.

\section{Conclusões}

1. Variações genéticas detectadas entre e dentro de procedências e progênies quanto à altura, DAP, volume, forma do fuste, árvores bifurcadas e árvores com copa quebrada revelam o potencial das populações em teste para ser explorado em termos de seleção.

2. As maiores produções volumétricas são apresentadas pelas procedências Guaimaca, Las Crucitas e San Marcos, originadas de regiões de menor longi- 
tude e latitude; San Luís e El Castaño apresentam a menor produção em volume e tem sua origem em região de maior longitude e latitude.

3. As correlações negativas entre a forma do fuste em Angatuba, SP, com as características geográficas de origem das sementes indicam que as procedências San Marcos, Guaimaca, Tablazon e Las Crucitas, originadas de regiões de menor longitude, apresentam árvores com fustes menos tortuosos.

4. A densidade básica da madeira e o índice de Runkel indicam que a madeira das procedências em teste em Angatuba, SP, terá alto rendimento volumétrico de celulose e fornecerá papel de boa qualidade.

5. A seleção de árvores visando maximizar o ganho genético em volume deve ser baseada no DAP, em virtude dos altos coeficientes de correlação genética aditiva seguido por baixos desvios-padrões entre essa característica com o volume.

\section{Referências}

AMARAL, A. C.; FERREIRA, M.; COUTO, H. T. Z. Métodos de avaliação da densidade básica da madeira de populações de pinheiros tropicais. IPEF, Piracicaba, v. 15, p. 47-67, 1977.

ARBUTHNOT, A. The influence of basic wood density of eucalypts on pulp and properties. In: IUFRO SYMPOSIUM ON INTENSIVE FORESTRY, 1991, Durban. Proceedings... Durban: International Union of Forest Research Organization, 1991. p. 966-975.

BARRICHELO, L. E. G. Caracterização de madeira de Pinus spp. IPEF, Piracicaba, v. 3, n. 14, p. 100-112, set. 1984.

FALCONER, O. S. Introdução à genética quantitativa. Viçosa, MG: UFV, 1987. 279 p.

KAGEYAMA, P. Y. Variação genética em progênies de uma população de Eucalyptus grandis Hill ex Maiden. 1980. 125 f. Tese (Doutorado) - Escola Superior de Agricultura Luiz de Queiroz, Piracicaba.

KAGEYAMA, P. Y.; VENCOVSKY, R. Variação genética em progênies de uma população de Eucalyptus grandis Hill ex Maiden. IPEF, Piracicaba, n. 24, p. 9-26, 1983.
MASSAKI, K. M. C. Variação entre espécies de procedências de pinheiros tropicais em Agudos, SP, Capão Bonito, SP, e Araquari, SC. 1989. 96 f. Dissertação (Mestrado) - Universidade Federal do Paraná, Curitiba.

MATZIRIS, D. I.; ZOBEL, B. J. Inheritance and correlations of juvenile characteristics in loblolly Pine. Silvae Genetica, Frankfurt, v. 21, p. 44-48, 1973.

RESENDE, M. D. V.; FERNANDES, J. S. C. Procedimento BLUP individual para delineamentos experimentais aplicados ao melhoramento florestal. Revista de Matemática e Estatística, Marília, v. 17, p. 87-109, 1999.

RESENDE, M. D. V.; HIGA, A. R. Estimação de valores genéticos no melhoramento de Eucalyptus: seleção em um caráter com base em informações do indivíduo e de seus parentes. Boletim de Pesquisas Florestais, Curitiba, n. 28/29, p. 11-35, jan./dez. 1994a.

RESENDE, M. D. V.; HIGA, A. R. Maximização da eficiência da seleção em testes de progênies de Eucalyptus através da utilização de todos os efeitos do modelo matemático. Boletim de Pesquisas Florestais, Curitiba, n. 28/29, p. 37-56, jan./dez. 1994 b.

RESENDE, M. D. V.; OLIVEIRA, E. B.; MELINSKI, L. C.; GOULART JUNIOR, F. S.; OAIDA, G. R. P. Seleção genética computadorizada: SELEGEN "best prediction": manual do usuário. Colombo: EmbrapaCNPF. 1994. $31 \mathrm{p}$.

SOUZA, S. M. de; RESENDE, M. D. V.; SILVA, H. D. da; HIGA, A. R. Variabilidade genética e interação genótipo $\mathrm{x}$ ambiente envolvendo procedências de Eucalyptus cloesiana F. Muell., em diferentes regiões do Brasil. Revista Árvore, Viçosa, MG, v. 16, n. 1, p. 1-17, 1992.

VENCOVSKY, R.; BARRIGA, P. Genética biométrica no fitomelhoramento. Ribeirão Preto: Sociedade Brasileira de Genética, 1992. 486 p.

WRIGTH, J. A. Introduction to forest genetics. New York: Academic, 1976. 463 p.

ZOBEL, B.; TALBERT, J. Applied forest tree improvement. New York: J. Wiley, 1984. 496 p. 